\title{
Un paysage institutionnel en recomposition : au-delà des structures, quelles perspectives pour la gouvernance territoriale? ${ }^{1}$
}

\author{
Marie-José Fortin \\ Chaire du Canada en développement régional et territorial \\ GRIDEQ/CRDT/UQAR \\ Marie-Joëlle Brassard \\ Recherche Action Innovation
}

\section{LA CRÉATION D'UN CONTEXTE D'AUSTÉRITÉ}

\section{Les coupes se succèdent dans plusieurs secteurs d'activités.}

L'automne 2014 est chaud au Québec! Le gouvernement Couillard, élu en avril, annonce qu'il mettra de l'ordre dans les finances publiques. L'heure est à l'austérité déclarée, avec en finalité le déficit zéro. Dès juin 2014, une Commission de révision des programmes est créée avec le mandat de mettre en place un processus d'évaluation continue de ses programmes. Aux vues du gouvernement, il faut recentrer là où les besoins sont les plus criants.

Les coupes se succèdent dans plusieurs secteurs d'activités; abolition des 18 agences de santé et de services sociaux, des 21 Conférences régionales des élus (CRÉ) et des Forums jeunesse, des 122 Centres locaux de développement, de certaines directions régionales, dont celles du ministère de l'Éducation et de l'Immigration et du Conseil du statut de la femme. Le gouvernement se veut rassurant, alléguant qu'en réduisant des structures trop coûteuses, les services aux citoyens seront préservés.

Pour le gouvernement, les interlocuteurs légitimes sont maintenant les élus municipaux, la municipalité régionale de comté (MRC) étant le palier pertinent de la «gouvernance de proximité ». C'est à cette échelle qu'il entend négocier une décentralisation des pouvoirs. Un pacte fiscal « tran- sitoire » était adopté à l'automne 2014, qui a fait l'objet de coupes à hauteur de 300 millions de dollars et un nouveau pacte fiscal est attendu pour 2016.

Du côté des grandes villes, et à la demande de l'Union des municipalités du Québec (UMQ), le gouvernement adoptait en décembre 2014 le projet de loi $3^{2}$ sur la réforme des régimes de retraite. La loi prévoit le refinancement des déficits passés des régimes de retraite à parts égales entre la partie patronale et les employés, déficits qui étaient sous la responsabilité des municipalités. La suspension de l'indexation automatique des pensions de retraite pendant une période de trois ans est également prévue, ce qui fait dire aux syndicats que cette situation appauvrira les retraités. L'enjeu financier est de taille et les chiffres contestés. Pour les années 2010, 2011 et 2012, la Régie des rentes du Québec (RRQ) évaluait le déficit à 3,9 milliards de dollars en février 2015. De son côté, le milieu syndical ${ }^{3}$ soutenait qu'il se situait entre 2,2 à 2,5 milliards de dollars, alléguant que le gouvernement avait gonflé les chiffres pour gagner la faveur de l'opinion publique. ${ }^{4}$

Le gouvernement se veut rassurant, alléguant qu'en réduisant des structures trop coûteuses, les services aux citoyens seront préservés.

\section{DES APPRENTISSAGES POUR CONSTRUIRE L'AVENIR?}

Ces éléments, en trame de fond, dressent une scène complexe où se jouent plusieurs négociations et enjeux en simultanée. Du point de vue du dévelop- pement régional, le contexte marque une rupture dans l'évolution des rapports entre l'État central et les acteurs des territoires. L'architecture institu- 
tionnelle construite sur 40 années d'évolution, articulée autour de la concertation régionale entre une multiplicité d'acteurs, est remise en cause au nom de la «gouvernance de proximité ».

Du point de vue des connaissances, cette période offre un contexte d'observation propice pour tester quelques énoncés théoriques qui justifient les fondements de l'action publique menée en développement régional. En particulier, les milieux innovateurs, régions apprenantes et économie de proximités expliquent les «régions qui gagnent» par les capacités de coordination de divers acteurs individuels et collectifs (Benko et Lipietz, 1992; Leloup et al. 2005; Camagni et al., 2004; Gilly et Torre, 2000). Une telle capacité serait construite dans le temps, sous deux angles: d'abord une proximité institutionnelle, où les acteurs partagent des représentations, conventions et règles communes et, en complément, une proximité organisationnelle, soit un «espace de définition des pratiques et des stratégies des agents à l'intérieur d'un ensemble de règles porté par les institutions » (Kirat et Lung, 1995, cités dans Bouba-Olga et al., $2008: 5)$.

Selon cette perspective, les capacités institutionnelles pourraient survivre à un changement organisationnel, comme l'abolition des Conférences régionales des élus (CRÉ) décrétée par le gouvernement. Mais si la structure organisationnelle était modifiée, et sachant qu'elle n'est pas sans incidence sur la façon de rendre une mission, quels effets et enjeux pourraient avoir une reconfiguration sur les dynamiques de gouvernance territoriale? Comme le soutiennent Torre et Beuret, la gouvernance est en effet un phénomène complexe et traversée de tensions : un « (...) ensemble des processus et dispositifs par lesquels des parties prenantes ou acteurs de différentes natures (productifs, associatifs, particuliers, représentants des pouvoirs publics ou des collectivités locales...) contribuent à l'élaboration, parfois concertée, parfois conflictuelle, de projets communs pour le développement futur des territoires » $(2012: 3)$.

Pour éclairer ces questions, nous avons mené une enquête auprès d'une quinzaine d'intervenants provenant de six CRÉ. Notre objectif était de cerner comment les acteurs territoriaux s'organisent face à ces changements : souhaitent-ils conserver de telles instances de concertation régionale? Si oui, selon quelles formes? Certaines pratiques seront-elles reconduites, dans d'autres instances? Nous faisions l'hypothèse qu'après des décennies d'expériences et malgré les difficultés propres à tout exercice de concertation, les acteurs territoriaux auraient appris à apprécier les fruits de la concertation régionale et construiraient un modèle inspiré par leurs pratiques. Pour reprendre les termes des théories évoquées précédemment, les apprentissages serviraient de levier pour fonder le paysage institutionnel en recomposition.

Mais cette hypothèse optimiste néglige l'influence de la stratégie gouvernementale et du contexte budgétaire particulier qui pèsent tous deux très lourd dans les processus de négociation. Quoiqu'exploratoire, l'enquête permet ainsi de révéler les tensions qui animent les discussions en cours quant aux orientations possibles pour l'avenir de la concertation régionale. Après en avoir décrit quatre, le modèle adopté dans deux CRÉ sera exposé. L'examen de son architecture révélera des enjeux importants qui risquent de se poser dans la gouvernance territoriale. Mais d'abord, posons un regard sur le travail réalisé par les CRÉ.

\section{Pour éclairer ces questions, nous avons mené une enquête auprès d'une quinzaine d'intervenants provenant de six CRÉ.}

\section{LES CONFÉRENCES RÉGIONALES DES ÉLUS}

Les Conférences régionales des élus ont été créées en 2003, en remplacement des Conseils régionaux de concertation et de développement (CRCD) fondés en 1992. Leur rôle était d'agir à titre d'interlocuteur privilégié du gouvernement en matière de développement régional. ${ }^{5}$ À ce mandat s'ajoutait initialement l'évaluation des organismes de planification et de développement local et régional et la production d'avis au ministre des Affaires municipales, des Régions et de l'Occupation du territoire, qui a cependant été contestée par les CRÉ. 
Le territoire couvert par les CRÉ correspondait aux régions administratives ${ }^{6}$. Leur conseil d'administration regroupait de 40 à 50 représentants composé des préfets des MRC, des maires de municipalités locales de 5000 habitants et plus et des villes dites de centralité, ainsi qu'un représentant par nation autochtone. Les membres de la société civile, nommés par les élus, composaient «au plus un tiers » du Conseil, une nette diminution par rapport aux défunts CRCD où ils constituaient les deux tiers des représentants avec droit de vote.

Le cadre de référence privilégié pour l'exercice de la concertation entre le gouvernement et les régions reposait sur le Plan quinquennal de développement (PQD) régional dans lequel s'inscrivaient les axes stratégiques de développement décidés par chaque région. À l'échelon infrarégional, les MRC devaient référer au PQD pour assurer une cohérence de leur vision avec l'échelon régional.

Partant des axes de développement identifiés dans le $\mathrm{PQD}$, des ententes spécifiques entre les régions (CRÉ) et le gouvernement central orientaient l'allocation du Fonds de développement régional (FDR) qui variait de 1,5 million à 2,3 millions de dollars selon les régions, en baisse dans les dernières années. Le Fonds couvrait le développement économique et l'emploi, la protection de l'environnement, le développement social, la culture, le loisir, le sport et l'éducation, la recherche et la science. Avec le temps, de nouvelles responsabilités furent déléguées par l'État aux CRÉ : la lutte contre la pauvreté et l'exclusion sociale, l'immigration, l'économie sociale avec les pôles, les Forums jeunesse, les aînés et la culture, les loisirs et le sport. Le mécanisme des ententes spécifiques permettait la rencontre d'organismes et de ministères régionaux autour d'un enjeu spécifique pour lever des montants d'argent supplémentaires. Selon les régions, les CRÉ pouvaient gérer annuellement des fonds se situant généralement entre 5 et 11 millions de dollars. ${ }^{7}$

Pour terminer, les CRÉ étaient responsables des Commissions régionales sur les ressources naturelles et le territoire (CRRNT). Elle devait élaborer et participer à la réalisation des plans régionaux de développement intégré des ressources naturelles et du territoire. L'exercice visait une vision cohérente pour le développement et la conservation des ressources naturelles et du territoire, qui était ensuite mis en œuvre par l'intermédiaire des Tables GIR, animées par les CRÉ.

Au fil des ans donc, le mandat des CRÉ s'est élargi, confirmant ainsi leur rôle d'interlocuteur privilégié du gouvernement en matière de développement régional.

\section{L'exercice visait une vision cohérente pour le développement et la conservation des ressources naturelles et du territoire, qui était ensuite mis en ouvre par \\ l’intermédiaire des Tables GIR, animées par les CRÉ.}

\section{QUATRE TENSIONS FONDATRICES D'UN MODÈLE DE CONCERTATION RÉGIONALE}

Les élus des CRÉ sont divisés suite à l'annonce du gouvernement d'abolir les CRÉ. Certains expriment publiquement être favorables à cette décision, comme le maire de Québec, président de la Communauté métropolitaine de la Capitale-Nationale, qui affichait déjà sa position en avril $2014^{8}$ : « on est rendu [avec la CRÉ] dans un mille-feuilles administratif qui est contre-productif $»^{9}$. D'autres sont au contraire soucieux de reconduire une telle instance jugée importante. Par exemple, dans une résolution adressée au gouvernement en ce sens, la CRÉ de la Mauricie précise: «[s]ans instance régionale pour assurer cette concertation, le risque est bien réel d'un retour rapide vers le travail en silo, retranché dans des réseaux limités, à l'échelle d'un territoire. $»^{10}$

Mais au-delà des positions et des déclarations publiques, que se dessine-t-il pour l'avenir de la concertation régionale? Au moment de recueillir les propos d'acteurs impliqués (avril, mai 2015), des négociations portent sur la cession de leurs activités se déroulent, selon les régions, au sein des conseils d'administration des CRÉ et/ou de comités de transition (formés des préfets des MRC). L'analyse des 15 entretiens révèle quatre tensions qui, selon les cas, incitent à adopter un modèle plutôt qu'un autre. 
Première tension: concurrence entre spécialistes et entre enjeux

L'ajout d'ententes spécifiques sectorielles a requis l'embauche de professionnels dans les CRÉ. En 2013, on comptait de 13 à 30 employés permanents selon les régions (tableau 1), ce qui va bien au-delà de l'équipe attitrée au mandat original de planification qui comptait moins d'une dizaine de personnes selon nos répondants. Ces spécialistes ont acquis des connaissances fines de leur territoire d'intervention, de ses enjeux et de la dynamique des acteurs présents. Dans certaines CRÉ, on dénotait un clivage entre des élus et leurs professionnels. De façon étonnante, l'expertise n'était pas forcément appréciée de certains élus. C'est du moins l'interprétation donnée lors d'entretiens pour expliquer entre autres les réactions plutôt tièdes des élus pour défendre leur organisation.

Sur un autre plan, celui de la gestion du temps et des priorités, les ententes spécifiques et les enjeux devenaient de plus en plus complexes et exigeants. Les élus devaient donc faire des choix entre différents dossiers. D'une part, ceux associés au palier local étaient estimés prioritaires parce c'est d'abord à cette échelle qu'ils sont imputables, en tant qu'élus municipaux. Cela est encore plus vrai pour les petites municipalités disposant de peu de ressources humaines spécialisées. D'autre part, les dossiers susceptibles de générer des retombées économiques mobilisaient davantage les élus et c'est pourquoi ils s'y investissaient davantage, estiment des répondants, dans les comités dits de développement économique.

Lorsque les enjeux étaient pas sexy, les élus n'étaient pas aux tables sectorielles. La pauvreté, les femmes, l'économie sociale, les dossiers à caractère social, c'était pas "glamour» pour eux. Les dossiers sexy c'était le transport, le développement économique, le développement forestier.

Tableau 1 - Nombre d'employés attachés aux CRÉ de chaque région du Québec (2012)

\begin{tabular}{|l|l|}
\hline $\begin{array}{l}\text { Conférence régionale des Élus (CRÉ) par région } \\
\text { administrative }\end{array}$ & $\begin{array}{l}\text { Emplois } \\
(\mathbf{2 0 1 2})\end{array}$ \\
\hline Bas-Saint-Laurent & 29 \\
\hline Saguenay-Lac-Saint-Jean & 19 \\
\hline Capitale Nationale & 25 \\
\hline Mauricie & 17 \\
\hline Estrie & 20 \\
\hline Montréal & 16 \\
\hline Outaouais & 17 \\
\hline Abitibi-Témiscamingue & 25 \\
\hline Côte-Nord & 25 \\
\hline Baie-James & 13 \\
\hline Gaspésie-les-Iles & 30 \\
\hline Chaudière-Appalaches & 14 \\
\hline Laval & 23 \\
\hline Lanaudière & 22 \\
\hline Laurentides & 20 \\
\hline Montérégie EST & 24 \\
\hline Agglomération de Longueuil & 16 \\
\hline Montérégie & 20 \\
\hline Vallée du Haut Saint-Laurent & 23 \\
\hline Centre-du-Québec & 19 \\
\hline Total & 417 \\
\hline & \\
\hline
\end{tabular}

Source : Rapports annuels des CRÉ pour l'année 2012-2013 


\section{Deuxième tension : le poids politique de la concertation ou de la division}

Après 20 ans de concertation dans les régions, les impacts positifs de l'approche concertée étaient établis. L'ajout de plusieurs ententes spécifiques, soutenues par les budgets afférents, démontrait l'efficacité du mécanisme mis en place. Euvrant à la territorialisation des politiques publiques, l'espace politique occupé par les CRÉ s'élargissait. Avec un budget à gérer de plus en plus important, estimé entre 5 et 11 millions de dollars par année selon les régions, ces quasis « gouvernements » régionaux étaient devenus un interlocuteur de plus en plus important pour l'État central.

Les régions étaient d'autant plus fortes lorsqu'elles parvenaient à parler d'une voie unie. C'est ainsi qu'avec les années, les dirigeants des CRÉ ont réalisé que les mêmes enjeux étaient rediscutés avec le gouvernement sans qu'il n'y ait de suivi. L'importance de coordonner plus efficacement leurs travaux et de faire connaître les priorités des régions auprès des ministères et organismes les ont conduits à se doter d'un regroupement en 2012. Ce nouvel espace commun cherchait aussi à contrebalancer la division traditionnelle du monde municipal autour des deux grandes organisations que sont l'Union des municipalités du Québec (UMQ) et la Fédération québécoise des municipalités (FQM).

\section{Troisième tension : la concertation sans conflit?}

Le modèle d'interactions institué entre les élus et le gouvernement au sein de la CRÉ valorise la concertation, au détriment d'une approche de revendications, voire conflictuelle. Les Forums jeunesse ont été rapidement confrontés à cette réalité dès 2007 alors que les jeunes questionnaient leur autonomie face à l'État. Un acteur aux premières loges de ce débat explique ainsi la scission entre le mouvement étudiant et les Forums jeunesse :

Depuis [2007], une logique de collaboration plutôt que de revendication s'est instituée au sein des Forum jeunesse. Ça nous coûte cher maintenant, parce que les Forums jeunesse sont aujourd'hui considérés comme des « collabo » et ils n'ont pas été soutenus lorsque le gouvernement a parlé de les abolir.

Se pose en filigrane la place réservée à la société civile et aux groupes plus critiques dans le modèle qui se dessine, déjà diminué avec le remplacement des CRCD par les CRÉ.

Selon plusieurs intervenants interrogés, les élus estiment que la revendication par la critique et encore plus le conflit sont à proscrire, car, selon eux, le gouvernement ne reconnaît pas les acteurs valorisant ce mode de négociation. Or, les représentants des associations et groupes sociaux jouent régulièrement dans ce registre. Se pose en filigrane la place réservée à la société civile et aux groupes plus critiques dans le modèle qui se dessine, déjà diminué avec le remplacement des CRCD par les CRÉ.

\section{Quatrième tension : la CRÉ et son environnement institutionnel}

Dans certaines régions, la CRÉ était devenue un acteur important, sollicité pour donner des avis sur divers dossiers, y compris dans les médias. Cela s'expliquerait particulièrement dans les régions comptant peu ou pas d'autres organisations en place, de même que par l'expertise acquise par la CRÉ dans des domaines variés : "Dans le désert, quel que soit la grosseur, un arbre est visible. Il y a peu d'organisations, peu de structures, ça fait en sorte que celle qui est là devient un joueur majeur parce qu'il n'y en a pas d'autres».

L'explication est reprise par plusieurs en référence à la position de la Ville de Québec. Selon eux, le gouvernement a surtout tendu l'oreille aux demandes de Québec et Montréal où, de fait, existent plusieurs instances de concertation, ce qui n'est pas forcément le cas dans les autres régions. En ce sens, les CRÉ ne joueraient pas le même rôle selon la région où elles s'inscrivent et la densité de son tissu institutionnel. 


\section{UN MODÈLE «VOLONTAIRE » AVEC UNE ARCHITECTURE À DEUX NIVEAUX}

\section{Les régions centrales étudiées ont convenu de la pertinence d'une concertation sans qu'il n'y ait, à notre connaissance, d'engagement concret, ni même de travail sur un éventuel modèle.}

Ainsi, même après 40 ans d'expérience de concertation régionale, les choix demeurent difficiles, basés sur des interprétations variées du travail d'organisme comme les CRÉ. Au moment de réaliser l'enquête, quelques régions élaboraient un modèle de gouvernance territoriale. Le maire de Montréal annonçait son intention de reprendre le modèle existant, alors que Québec s'était limité à un «forum des élus » aminci, excluant d'autres types d'acteurs, dont la société civile ou même les experts professionnels. Les régions centrales étudiées ont convenu de la pertinence d'une concertation sans qu'il n'y ait, à notre connaissance, d'engagement concret, ni même de travail sur un éventuel modèle. Finalement, deux régions périphériques ont été à pied d'œuvre et nous ont transmis un modèle.

Les propositions élaborées ne sont pas définitives, mais des options se dessinent qui ont fait l'objet d'un accord au sein de l'exécutif. Les organigrammes construits présentent une architecture similaire (tableau no 2). Dans les deux cas, la gouvernance est organisée autour de deux niveaux jouant des rôles différents.

À un premier niveau, un forum régional rassemble des élus et des représentants de la société civile qui déterminent les grands enjeux de planification stratégique. Les ressources financières et professionnelles requises pour mener un tel exercice découlent d'un montant relié à l'adhésion des MRC participantes. À un second niveau, se déroule la mise en œuvre des projets avec un financement qui provient des MRC participantes sur une base volontaire. Ainsi, les MRC font le choix d'investir dans certains enjeux et projets estimés importants. En conséquence, le financement permettra de rémunérer les professionnels qui y travailleront. Il est cependant attendu que l'équipe permanente sera très restreinte, les spécialistes entrant et sortant au gré de mandats, voire contrats accordés par les MRC. Quant à la société civile, elle joue un rôle consultatif aux deux niveaux. Ses représentants groupes sont invités à s'impliquer mais sans pouvoir décisionnel.

\section{Tableau 2 - Modèle de gouvernance retenu dans deux régions}

\section{Niveau régional}

- Mission de planification : prioriser les enjeux régionaux

- Élus des MRC avec pouvoir décisionnel

- Société civile avec pouvoir consultatif

\section{Niveau infra-régional \\ Gestion de projets sur la base d'intérêts communs des MRC}

- Mission de mise en œuvre d'initiatives concrètes : répondre aux enjeux considérés prioritaires par des MRC qui les investissent sur une base volontaire

- Ressources: financement à partir d'une cotisation volontaire des MRC participantes à un projet

- Ressources : Financement de professionnels par les MRC impliquées

- Élus des MRC participantes avec rôle décisionnel

- Représentants de la société civile concernés par le sujet, avec rôle consultatif 


\section{QUELS ENJEUX POUR LA GOUVERNANCE TERRITORIALE?}

La recherche fut réalisée dans un contexte d'incertitudes. Le contexte soulève des tensions et renforce la complexité de la gouvernance territoriale. Or, le modèle retenu dans deux CRÉ prolonge des tendances déjà observées, et qui sont de plus en plus présentes avec la succession de réformes observées depuis 30 ans. Deux caractéristiques méritent d'être soulignées en ce sens, car elles apparaissent à contresens de la définition précédente de la gouvernance.

\section{Une gouvernance centrée sur les élus}

Le modèle mise fondamentalement sur les élus et le palier mercéen. C'est à ce niveau que se déroule la mise en œuvre, que sont accumulées les ressources financières et humaines, et où se prennent les décisions, par les élus locaux. Déjà, ils sont les seuls invités à décider ou à négocier certaines priorités et orientations (ex. : suite des CLD, pacte fiscal, fermeture des CRÉ) ${ }^{11}$.

Certes, cette échelle infra-régionale se rapproche davantage de l'échelon local qui correspond à leur première appartenance et à leur imputabilité en tant qu'élu municipal. C'est pourquoi nombre d'élus semblent favorables à un délaissement de l'échelle régionale au profit de celui de la MRC. Notons cependant que certains risquent d'avoir plus d'influence que d'autres, selon leur rôle ou la réalité qu'ils assument. Diriger une ville ou une MRC avec des ressources financières et humaines donne des capacités que la plupart des élus ruraux n'ont pas. De même, associer la gouvernance régionale à l'imputabilité des élus néglige les rôles de coordination, de mobilisation des ressources et de territorialisation des politiques publiques joués par les institutions comme les CRÉ pour articuler les rapports entre les régions et l'État.

Enfin, comme les orientations du gouvernement s'inscrivent dans la continuité des changements amorcés avec la création des CRÉ, la place de la société civile apparaît de plus en plus congrue, au bénéfice des élus locaux. L'application du modèle construit dans les deux régions permettra de mesurer la place de la participation des membres de la société civile selon les enjeux et les projets.

\section{Comme les orientations du gouvernement s'inscrivent dans la continuité des changements amorcés avec la création des CRÉ, la place de la société civile apparaît de plus en plus congrue, au bénéfice des élus locaux.}

\section{Des projets « à la carte »}

À la différence des ententes spécifiques s'appliquant à l'ensemble d'une région, les projets sont mis en œuvre sur une base volontaire, deuxième caractéristique du modèle. Articulé en deux niveaux, celui de la concertation régionale impliquant toutes les MRC sera probablement plus facile à réaliser. En effet, le diagnostic des enjeux fait généralement consensus. C'est davantage la conception et la mise en œuvre de stratégies d'actions pour y faire face, décidées au second niveau, que les décisions risquent d'être les plus déchirantes. Or, ici, le changement est important par rapport aux pratiques passées, car, à ce niveau, c'est la capacité de payer qui risque de devenir un, sinon le facteur clé pour qu'une MRC puisse s'impliquer dans un chantier.

La gestion «par projets », lire à la pièce, est-elle une porte ouverte pour reconduire au secteur public la logique du modèle entrepreneurial propre à l'entreprise privée? Et quels seraient les effets à moyen et long termes de cette logique de projets en contexte d' « austérité » et de moyens restreints : est-il probable que certains enjeux économiques susceptibles de générer des revenus soient plus investis par rapport à ceux s'attaquant à des problèmes sociaux dont les bénéfices sont souvent visibles à plus long terme? Risque-t-on de voir des enjeux « orphelins »(transport, environnement, aînés, lutte contre la pauvreté, apprentissages à la démocratie par les jeunes)? Enfin, observera-t-on un accroissement des écarts entre les MRC ayant la capacité de payer et s'investir dans des projets et celles qui ne les ont pas? Les possibles scissions entre mission économique et mission sociale, ainsi que la question classique des disparités représentent de vrais enjeux potentiels dans ce modèle. 


\section{CONCLUSION : LA FIN DE LA SOLIDARITÉ RÉGIONALE?}

Depuis l'automne 2014, le gouvernement Couillard associe dans une même dynamique « austérité », «crise budgétaire » et « décentralisation ». La stratégie gouvernementale joue sur deux fronts à la fois : d'un côté, des ressources sont rapprochées du local et des municipalités, via les MRC, de l'autre, il y a une recentralisation de services et personnel de directions régionales vers les ministères. Mais dans les deux cas, les ressources sont diminuées et le palier régional en ressort considérablement affaibli.

\section{La dynamique actuelle fabriquée par le gouvernement teste aussi la capacité des acteurs territoriaux à se concerter, à se fédérer.}

De leur côté, les élus locaux attendent un retour d'ascenseur de la part du gouvernement pour compenser leurs efforts suite à l'adoption du pacte fiscal transitoire 2014-2015 qui les amputait de 300 millions de dollars. L'attente d'un pacte fiscal annoncé en 2016 incite à tempérer, voire à évacuer un rapport conflictuel entre les municipalités et l'État. Or, comme l'expliquent Torre et Beuret (2012: 73), la gouvernance est un mouvement continu entre contestation et participation, entre opposition et coopération, «deux tendances opposées, dont la synthèse conduit à la définition des projets de développement et contribue à la définition des sentiers de développement des territoires». Serions-nous devant une nouvelle utopie qui consisterait à penser le développement des régions comme étant apolitique, sans qu'il n'y ait de divergences d'intérêts, sans tensions entre les acteurs?

La dynamique actuelle fabriquée par le gouvernement teste aussi la capacité des acteurs territoriaux à se concerter, à se fédérer. Ce sont les rapports entre l'État et les régions qui se transforment. De «partenaire » et «accompagnateur », on semble passer à l'«État entrepreneur» qui soutient d'abord les territoires aptes à se mobiliser, « innovants » et les « régions qui gagnent ». Et les premières réponses des élus, quoiqu'encore embryonnaires, vont sommes toute dans le sens des orientations $\mathrm{du}$ gouvernement valorisant une échelle de proximité. Ces orientations soulèvent plusieurs enjeux. En effet, selon les modèles qui se dessinent, les MRC souffrant le plus de problèmes de dévitalisation et disposant de moyens financiers précaires parviendraient difficilement à être parties prenantes de chantiers de développement structurants. L'abolition des chantiers régionaux menés par les CRÉ sonnerait-elle la fin de la solidarité régionale, au cour des ambitions historiques des politiques de développement régional? Le prix serait lourd à payer au nom de l'autonomie des territoires. Bref, si les CRÉ avaient leurs limites, toute architecture organisationnelle soulève son lot d'enjeux qui risquent d'être plus visibles dans le paysage de demain.

\section{BIBLIOGRAPHIE}

Benko, G et Lipietz, A. (dir.) (1992) Les Régions qui gagnent: districts et réseaux: les nouveaux paradigmes de la géographie économique, Paris : Presses universitaires de France, p. 35 à 55.

Bouba-Olga, Olivier et al (2008) «Avant-propos », Revue d'Économie Régionale \& Urbaine no 3 :279-287. DOI 10.3917/reru.083.0279.

Camagni, R., D. Maillat et A. Matteaccioli (dir.) (2004)

Ressources naturelles et culturelles, milieux et développement local. Institut de recherches économiques et régionales de Neuchâtel, Éditions ÉDES, $298 \mathrm{p}$.

Leloup, F., L. Moyart et B. Pecqueur (2005) «La gouvernance territoriale comme nouveau mode de coordination territoriale? », Géographie, économie, société, Vol. 7 (4) : 321-332.

Gilly, Jean-Pierre et A. Torre (dir.) (2000) Dynamiques de proximité. Paris: L'Harmattan, collection Emploi, Industrie et Territoire. $301 \mathrm{p}$.

Torre, André et Jean-Eudes Beuret (2012) Proximités territoriales. Éditions Économica, antropos, Paris, 105 p.

\section{NOTES}

${ }^{1}$ Ce texte a fait l'objet d'une présentation lors du colloque «L'innovation sociale en période d'austérité » organisé par le CRISES, au congrès de l'ACFAS, le 26 mai 2015. Il sera publié dans un numéro de la revue Organisations et territoires, prévu pour l'automne 2015.

Pour en citer un extrait : Fortin, Marie-José et Marie-Joëlle Brassard (2015) «Un paysage institutionnel en recomposition : au-delà des structures, quelles perspectives pour la gouvernance territoriale? », Organisations et territoires (à paraître).

${ }^{2}$ Devenue la loi 15 après son adoption. 
${ }^{3}$ La Coalition syndicale, représentée par monsieur Marc Ranger du Syndicat canadien de la fonction publique (SCFP).

${ }^{4}$ Fillion, Gérald (11 février 2015). Radio Canada Info, Blogue de Gérald Fillion, 3,9 milliards \$ ou 2,6 milliards \$? http://blogues.radio-canada.ca/geraldfillion/2015/02/11/39milliards-ou-26-milliards/

${ }^{5}$ Les informations sont tirées du site du ministère http://www.mamrot.gouv.qc.ca/amenagement-du-

territoire/guide-la-prise-de-decision-en-urbanisme/acteurset-processus/conference-regionale-des-elus/

${ }^{6}$ Certaines régions ont fonctionné sur la base d'un découpage à plus petite échelle, comme c'est le cas de la Montérégie divisée en trois territoires.

${ }^{7}$ En référence aux rapports annuels des CRÉ 2012-2013.

${ }^{8} \mathrm{http}: / / w w w . j o u r n a l d e q u e b e c . c o m / 2014 / 04 / 24 /$ labeaumereclame-de-nouveau-labolition-de-la-cre-de-la-capitalenationale

${ }^{9} \mathrm{http} / / / \mathrm{www} . q u e b e c h e b d o . c o m / A c t u a l i t e s / P o l i t i q u e / 2014-$ 02-18/article-3620313/Abolition-exigee-de-la-Conferenceregionale-des-elus-de-Quebec/1

${ }^{10} \mathrm{http}: / /$ www.cre-mauricie.qc.ca/communiques/2014/11/lacre-de-la-mauricie-demande-le-maintien-d-une-instanceregionale-de-concertation-et-de-developpement/93184 (consultée le 22 juin 2015)

${ }^{11}$ «Cette nouvelle étape vers une gouvernance des élus prévoit entre autres l'abolition des Conférences régionales des élus et le transfert de leurs responsabilités aux MRC. » - Pierre Moreau, ministre des Affaires municipales (http://ici.radio-canada.ca/regions/abitibi/2014/11/06/001abolition-cre-conference-regionale-des-elus-reactionscreat-abitibi-temiscamingue.shtml consultée le 22 juin 2015 


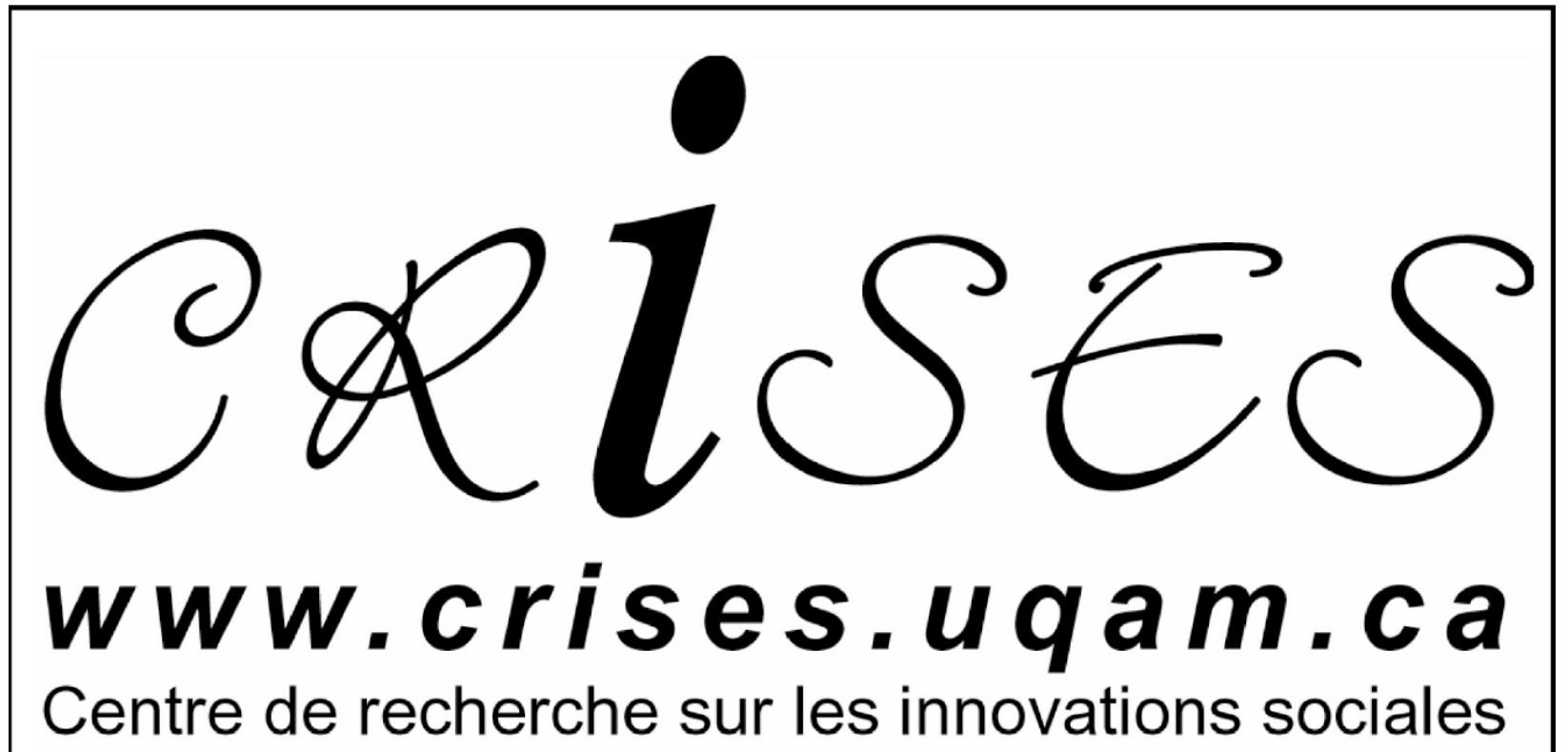

\section{Le Centre de recherche sur les innovations sociales \\ Une organisation interuniversitaire et pluridisciplinaire qui étudie et analyse les innovations et les transformations sociales}

\section{Objectifs :}

- Offrir un lieu de concertation et de coordination pour les activités de recherche

- Favoriser l'émergence de nouvelles pistes de recherche fondamentale et appliquée

- Développer de nouveaux partenariats

- Organiser des activités scientifiques et de transferts vers la communauté

- Former de jeunes chercheurs

\section{Renseignements :}

crises@uqam.ca

www.crises.uqam.ca

(514) 987-3000, poste 4458 